\title{
SUDDEN CARDIAC ARREST AND A PROBLEM IN TOPOLOGY
}

\author{
SIDNEY A. MORRIS and PETER J. NYIKOS
}

(Received 22 June 1989; revised 19 December 1990)

\begin{abstract}
In the May 1983 issue of Scientific American, A.T. Winfree published an article which gave an explanation of sudden cardiac arrest in terms of topology. His topological argument was modified in his 1987 book called "When time breaks down". In this paper we fill what appears to be a gap in Winfree's topological arguments.
\end{abstract}

Sudden cardiac arrest is a leading cause of death. Experiments carried out at the beginning of this century, notably by G.R. Mines (see [2], [3]) showed how even healthy hearts can be made to undergo fibrillation by relatively weak electrical shocks or neural stimuli delivered at certain fleeting instants (generally about 30 milliseconds long) in the heartbeat cycle.

In [2] and [3], Arthur T. Winfree gives a topological explanation for this phenomenon, based upon two essentially different ways in which the timing of the heartbeat is affected by stimuli. One way is called weak rescheduling in [2] and odd (or weak) resetting in [3]. As defined in [2] and [3] its essential property is that, as the termination point of the stimulus is varied continuously through one cycle, the time to the beginning of the next cycle passes continuously through the full range, from the cycle length to zero. The other way, called strong rescheduling in [2] and even (or strong) resetting in [3], has the property that the time to the beginning of the next cycle does not pass through the full range of values.

As the name implies, weak resetting is elicited by relatively weak stimuli, with zero stimulus as the limiting case. In between these stimuli and those

\footnotetext{
${ }^{1}$ The University of New England, Armidale, N.S.W. 2351, Australia.

${ }^{2}$ University of South Carolina, Columbia, SC 29208, U.S.A.

(C) Copyright Australian Mathematical Society 1991, Serial-fee code 0334-2700/91
} 
eliciting strong rescheduling, Winfree's topological argument implies the existence of a stimulus which, when applied at some critical instant, has an inherently unpredictable effect on the timing of the next beat.

Winfree and others have, in more technical articles, generalised the concepts of weak and strong rescheduling using the concept of winding number and the fact that functions of different winding number are homotopicaly inequivalent. We shall outline a short proof of Winfree's result in this spirit for topologically sophisticated readers.

The purpose of this article is to show how Winfree's argument in [3] (though not, apparently, in [2]) can be filled out using concepts accessible to anyone with a strong calculus background. In the first proof we shall use Tietze's extension theorem, in the following simple form: a continuous realvalued function from a closed, bounded subset of the Euclidean plane can be extended to a continuous function defined on the entire plane. Later we shall give a method of constructing such an extension for the specific subset of the plane needed for Winfree's argument, which is self-contained and uses only facts mentioned in standard calculus texts, and proved in advanced calculus. The only advanced theorem remaining in the resulting argument is the "no retraction" theorem already used in the part of the argument found in [3].

Following Winfree, we plot the time before the following beat as a function on a rectangle $R$.

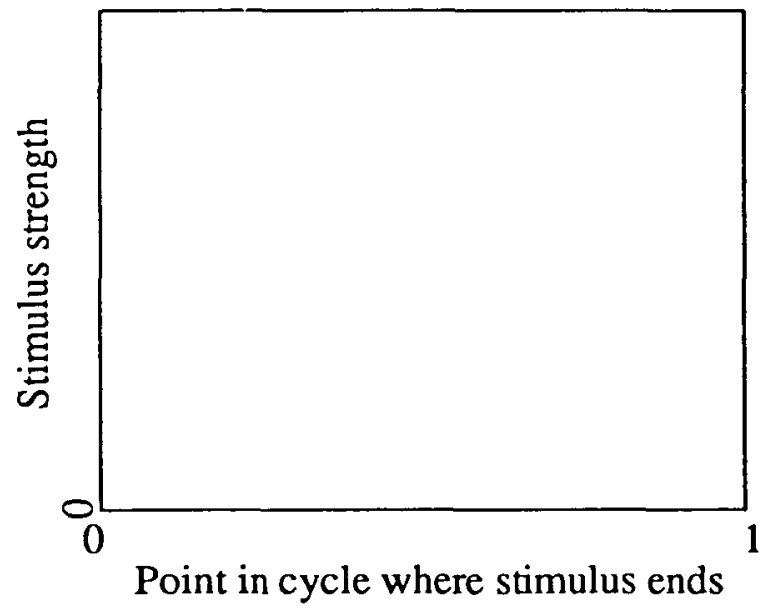

The bottom edge ("floor") of $R$ represents zero stimulus strength; the top edge ("roof") represents a strength which produces even resetting. The vertical sides ("walls") represent stimuli applied at the beginning, and, which amounts to the same thing, the end, of a cycle. Functional values of the corresponding points of the left and right walls are thus the same. The length of 
a single cycle is scaled to make it equal 1 , and times greater than or equal to 1 are reduced modulo 1 ; that is, we subtract the integer part of any number $\geq 1$ to produce a number in the interval $[0,1)$.

The main theorem is that it is impossible to extend this function continuously throughout the region $R$. [If there were a discontinuity on $R$, there would be nothing to prove; however, the beginning of the cycle is chosen so as not to be one of the critical points in the cycle where a stimulus of intermediate strength is disruptive.] The physical implications of this impossiblity are explored in [2] and [3].

We now abstract the mathematical essentials of the function. Other examples of functions satisfying the given conditions appear in [2] and [3], relevant to a variety of interesting medical, biological, chemical, and physical phenomena.

Definition. A colouring of a subset $S$ of the plane $E^{2}$ is a function $f$ from $S$ into $[0,1]$. A colouring is said to be continuous if $f$ is continuous as a function into $[0,1]$ with the endpoints identified.

Informally, 0 and 1 are taken to be the same colour ("red") and the identification space of $[0,1]$, which is topologically a circle, is thought of as a continuous spectrum, with violet close to 0 and orange close to 1 before the identification of the endpoints is made. So we shall refer to $[0,1]$ with endpoints identified as "the spectrum", and its elements (numbers modulo 1) as "colours".

The continuous colouring of $R$ satisfies the following conditions:

1. "The full cycle of colours is encountered on the bottom edge of the rectangle". More precisely, the colouring $f$ of $R$ takes on every value in $[0,1]$ exactly once on the bottom of the rectangle, except that the two corners are given the same value.

2. "The left and right walls of the rectangle are identically coloured." More precisely, points on the same horizontal line are given the same value of $f$. In particular, this is true of the two top corners.

3. There are colours missing from the "roof" (top edge). In other words, $f$ takes the roof to a proper subset of the spectrum, which by continuity is a closed subinterval of the spectrum, and topologically the same as a closed interval $[a, b]$ of the line.

Given these conditions on $f$, we have:

THEOREM. There is no continuous extension of $f$ to the region enclosed by $R$. 
To prove this, let $C$ be a circle in the plane which surrounds and nowhere touches $R$, and let $g$ be a continuous colouring of $C$ which maps $C$ oneto-one onto the spectrum.

The following key result is implicit in [3]. However no formal proof is given. (See p. 63 of [3] for a discussion of the result.)

MAIN LEMMA. The colourings $f$ and $g$ can be extended simultaneously to a continuous colouring of the annular region between $R$ and $C$.

This lemma, together with the "no retraction" theorem of topology [3, Chapter XVI Corollary 2.2], yields the theorem. Indeed, as pointed out on p. 63, such a continuous extension would, by the lemma, result in a continuous extension of $g$ to the entire disk $D$ bounded by $C$. But then we could define a function $h$ from $D$ to $C$ by simply sending each point of $D$ to the unique point of $C$ which receives the same colour. The colouring being continuous, the function $h$ is also continuous, violating the "no retraction" theorem.

First PROOF OF MAIN LemMa. Draw simple smooth arcs $A_{0}$ and $A_{1}$ from the two lower corners of the rectangle $R$ to the point on $C$ which is given the same colour, without $A_{0}$ or $A_{1}$ touching each other or intersecting $R$ or $C$ anywhere else.

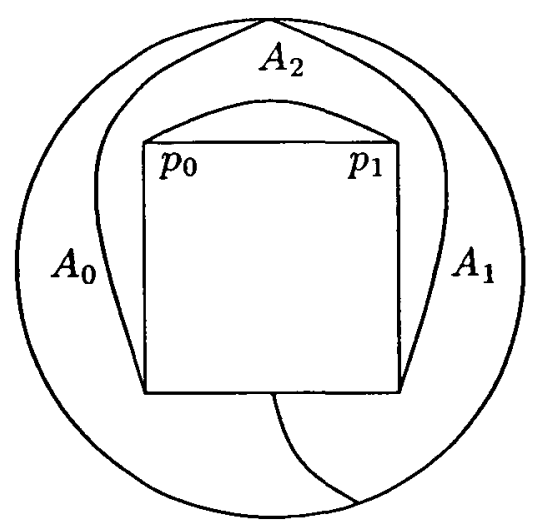

Points elsewhere on the circle are similarly joined to the corresponding points on the bottom edge of $R$ by arcs in a continuously varying fashion, no two arcs meeting, and no arc touching either $R$ or $C$ anywhere else; the arcs are also to fill the region between $C$ and the union of $A_{0}$ and $A_{1}$ with the bottom edge of $R$. (For details, see Remark 1.) Similarly, it is easy to connect the two top corners $p_{0}$ and $p_{1}$ of $R$ by an arc $A_{2}$ that stays inside 
the region bounded by the union of $A_{0}$ and $A_{1}$ with the three other edges of $R$, and to fill in the region bounded by $A_{0}, A_{1}, A_{2}$ and the "walls" of $R$ by nonintersecting arcs that connect the corresponding points of the left and right "walls" of $R$. By taking all points on a constructed arc to the same colour, we thus extend the union of $f$ and $g$ to all of the annular region between $R$ and $C$ except for the interior of the closed region $L$ whose boundary $B$ is the union of $A_{2}$ with the upper edge of $R$ :

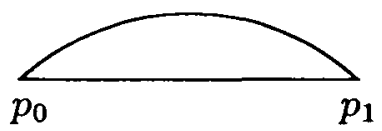

Now $L$ is a metric space, and $B$ is a closed subspace. By Condition 3 on $f$, and the construction of the extension above [all of $A_{2}$, including $p_{0}$ and $p_{1}$, is sent to the same colour], $B$ has been taken continuously to a topological copy $A$ of $[a, b]$. By Tietze's extension theorem, [3, p. 149] there is a continuous extension of this map to all of $L$, with range $A$. This completes our first proof of the Main Lemma.

Alternative PROOF OF THEOREM. For those familiar with homotopy theory, here is a short alternative proof which does not require the main lemma. Suppose $f$ could be extended to a continuous function $g$ on all of the region enclosed by $R$. By change of scale and translation, we may assume this region is the unit square $[0,1] \times[0,1]$. For each point $(x, y)$ in the region, let $h(x, y)=g(x, y)-g(0, y) \bmod 1$. This means that we subtract the nearest integer $\leq$ our figure, so that $h(x, y)$ is in the interval $[0,1)$. Then $h$ is identically zero on both walls, and is a continuous colouring. Now take 0 to be the basepoint in the spectrum. The function $h$ can be regarded as a homotopy leaving this basepoint fixed: the homotopy is between the map $h_{0}$ taking $x$ to $h(x, 0)$ and $h_{1}$ taking $x$ to $h(x, 1)$. But the homotopy class of $h_{0}$ generates the fundamental group of the circle, which is infinite cyclic. On the other hand, since the range of $h_{1}$ is a proper subset of the spectrum, its homotopy class is the identity element of this group, contradicting the existence of a homotopy between $h_{0}$ and $h_{1}$.

REMARK 1. The filling in of the annular region between $R \cup L$ and $C$ can be done by an induction as in Urysohn's lemma [3, p. 146]. Take the region $G$ bordered by $C, A_{0}, A_{1}$, and the bottom of $R$. An arc $A(1 / 2)$ joining the points "coloured $1 / 2$ " can be defined to divide $G$ into two subregions; then arcs $A(1 / 4)$ and $A(3 / 4)$, similarly defined, can divide each of these into two subregions; and so we continue with binary subdivisions, using dyadic 
rationals, i.e. numbers of the form $k / 2^{n}$, with $k$ an integer between 0 and $2^{n}$. In this way an infinite family of nonintersecting arcs is defined. Now at each stage $n$ in the induction, any point $p$ of $G$ is either on an arc, in which case its colour is already determined; or it is on a subregion between arcs representing $k_{n} / 2^{n}$ and $\left(k_{n}+1\right) / 2^{n}$, where $k_{n}$ depends on $p$ as well as $n$. If $p$ is never on a constructed arc, it is assigned the value $\lim _{n \rightarrow \infty} k_{n} / 2^{n}$, which exists. Continuity follows from the fact that we arrive at the same value for the limit no matter what direction $p$ is approached from.

Thus we have extended $f$ to the entire region outside $L$. If, in addition, our arcs are drawn evenly enough so that (a) the distance between arcs goes to zero as the difference between their colours goes to zero and (b) the straightline distance between points on the same arc increases monotonically with the distance along the arcs, then it can be shown that outside $L$ every colour occurs on a family of disjoint arcs.

REMARK 2. The existence of a continuous extension from $B$ to $L$ (last paragraph of the first proof of the Main Lemma) was explained on p.63 of [3] by saying "the roof, consisting only of even-resetting segments along which hue changes undirectionally then falls back again, connects to itself through concentric arcs of colour". This seems to presuppose more than the three conditions given, but if one does not take the word "concentric" too literally, an argument like that in Remark 1 is still possible. Here is a more elementary argument, promised in the introduction, for extending from $B$ to all of $L$.

Let $A_{2}$ be an arc of a circle of radius $M$. Consider the graph of the restriction of $f$ to the roof, with the roof represented by the horizontal coordinates and the colours represented by the vertical coordinates. The horizontal line at $f\left(p_{0}\right)=f\left(p_{1}\right)$ will be used as a reference line; the regions between it and the graph of $f$ (shaded below for a sample function $f$ ) are used as a guide to the defining of the "concentric arcs".

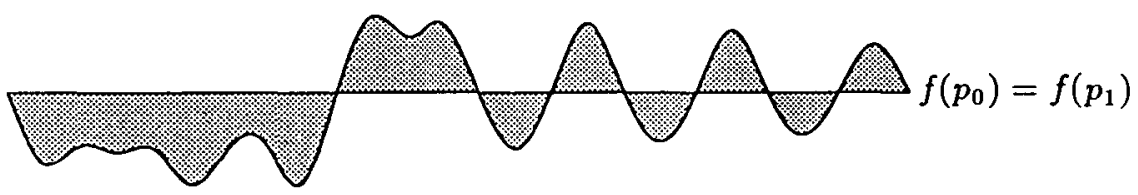

Here is how to do it. Each horizontal line that meets the graph represents a single colour. With most of the functions encountered in calculus and physics, such a horizontal line meets the shaded region in a finite family of disjoint closed intervals, some degenerate; that is, single points. For more 
general continuous functions it is more convenient to study the interiors of the regions, specifically excluding the graph of $f$. Each horizontal line meets the interior in a (possibly empty) family of disjoint open intervals. As the horizontal line moves either up from $f\left(p_{0}\right)=f\left(p_{1}\right)$ or down, the total length of these intervals decreases monotonically. In fact, if $f\left(p_{0}\right) \leq a_{0}<a_{1}$, each interval associated with $a_{1}$ projects to a portion of some open interval associated with $a_{0}$ (see illustration); similarly if $a_{1}<a_{0} \leq f\left(p_{0}\right)$.

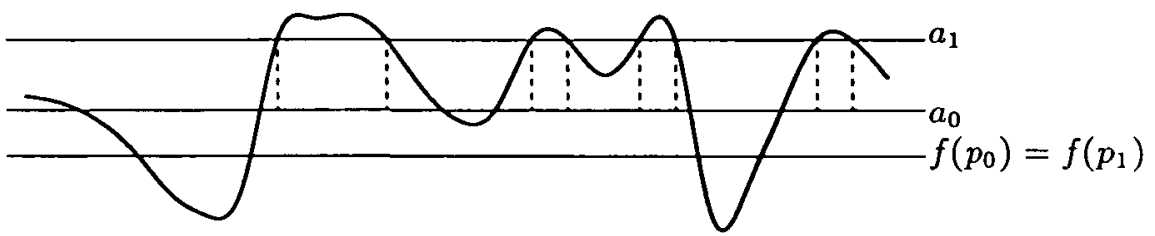

The trick is now to join any two points of the roof by an arc of a circle of radius $M$ if they correspond to the abscissae of the endpoints of an open interval obtained as above. In particular, they will have the same colour (determined by the horizontal line whose subinterval we are using). The arc with which we join them will not meet any other arc we construct, except in the special case of points with colour $f\left(p_{0}\right)=f\left(p_{1}\right)$, where two arcs may meet at their endpoints.

Now there will generally be some points of $L$ which are not on any arc constructed as above. For instance, suppose there is a local minimum above $f\left(p_{0}\right)$ as shown:

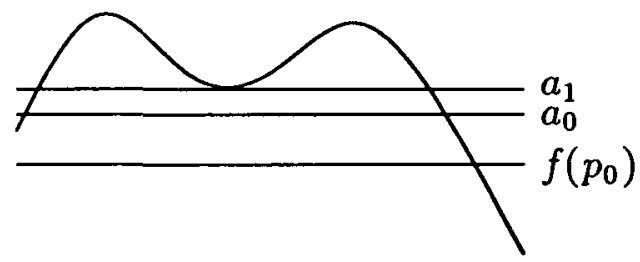

If this point is on the horizontal line with ordinate $a_{1}$, then the two arcs we associate with the illustrated intervals will look somewhat like this:

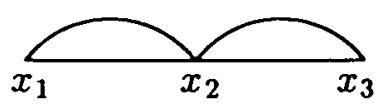

On the other hand, the two subintervals project to a single interval for any number $a_{0}$ in interval $\left[f\left(p_{0}\right), a_{1}\right)$, and the arcs resulting from these 
single intervals converge to an arc joining $x_{1}$ and $x_{3}$ as $a_{0}$ converges to $a_{1}$, leaving a region $R_{0}$ in which there are no constructed arcs:

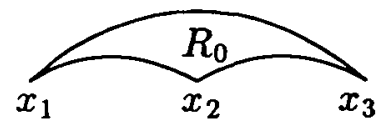

The simplest solution is to colour the entire region with the (single) colour of the points $x_{1}, x_{2}, x_{3}$.

Another possible region is between $A_{2}$ and the other arcs (if any) associated with $f\left(p_{0}\right)=f\left(p_{1}\right)$.

There are more complicated ways in which such regions can arise but in every case the simple solution of using a single colour works. If one desires every colour to appear only on a union of arcs ("isochronal lines") this can also be done, by just giving the boundary the single colour and then using a simple "target with bull's-eye" system of contours inside the region.

The general way to identify and isolate such regions is to look at the intervals that pass through an arbitrary fixed vertical line:

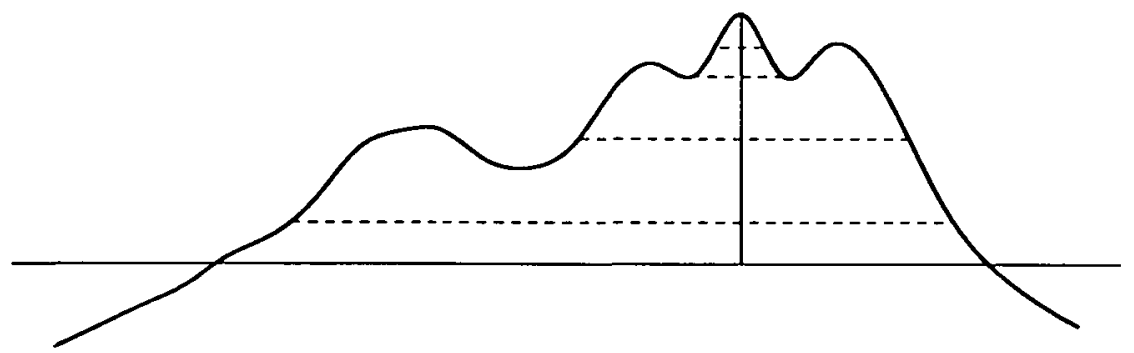

If the graph is above $f\left(p_{0}\right)$ on this line (as shown), we push a horizontal line up and observe the interval inside the region that crosses the chosen vertical line. The interval may shrink continuously for a while, in which case the arcs we are defining also shrink continuously and the space between them is filled up. But at some points the shrinking may experience a jump discontinuity, and here is where regions like $R_{0}$ will arise. By identifying all such discontinuities over all vertical lines [it is enough to consider the vertical lines with rational abscissae] we locate all the regions that require special attention.

REMARK 3. In the Scientific American article [2] a different proof for the main theorem was claimed: it was said that if one had a continuous extension of $f$ to the entire interior of the rectangle $R$, then a circle $C^{\prime}$ (topologically speaking: a simple closed curve) could be drawn in the interior such that 
every colour appears on $C^{\prime}$ exactly once. A fully general proof of this sort must, of necessity, use the false claim of the existence of such an extension, hence the above proofs are preferable. If one removes a point $p$ from the interior, then $f$ can easily be extended to the resulting punctured rectangular region without any such $C^{\prime}$ existing (except, of course, in the degenerate case where the roof and two walls all receive a single colour): just connect each point $r$ of $R$ to $p$ by the straight line segment whose endpoints are $r$ and $p$, and give the segment the same colour you gave $p$. Now on any simple closed curve enclosing $p$, the colour appears at least as often as it does on $R$.

Admittedly, the way of extending $f$ outlined here is not very faithful to the physical phenomena from which $f$ arose, so some extra hypotheses might yield such a $C^{\prime}$. For instance, the examples illustrated in either [1] or [2] seem to have such circles $C^{\prime}$. In particular, the boundaries of the "black holes" to which the colouring is not extended, and which together contain the set $Z$ of all points where behaviour is inherently unpredictable, all appear to be simple closed curves where each colour appears at exactly one point. Mathematically speaking however, even if such circles $C^{\prime}$ do appear, there is no reason why the boundary of the set $Z$ should have a simple colouring even if $Z$ is a simple region; indeed, one need consider only the case where the circle $C^{\prime}$ is the circle $C$ of the Main Lemma, which in turn is in the interior of a larger rectangle, while $Z$ is the interior of the rectangle $R$ of the Main Lemma.

This mathematical observation may be relevant to some speculation on pp. 157-159 of [1] of how fibrillation might start. There, some experiments are cited in which circular waves ("rotors") are observed on the surface of heart muscle. Were the latency at each point on the muscle plotted at a given instant in time, these waves would correspond to a circle $C^{\prime}$ as above. It is hypothesised in [1] that if such a $C^{\prime}$ were to border a region of degenerate tissue (the analogue of the set $Z$ ) then the rotor might "shatter" and thus initiate fibrillation. On the other hand, in line with the observation in the preceding paragraph, there might be a buffer zone between $C^{\prime}$ and $Z$ in which behaviour is continuous but not circulatory. Which of these two possibilities is more conducive to fibrillation? Which is more often found in nature?

\section{Acknowledgements}

The authors wish to thank Graham Elton for a useful discussion and Arthur Winfree for providing reference material. 


\section{References}

[1] J. Dugundji, Topology (Allyn and Bacon, Boston, 1966).

[2] Arthur T. Winfree, “ Sudden cardiac death: a problem in topology", Scientific American 248 (1983), 144-161.

[3] Arthur T. Winfree, When time breaks down (Princeton University Press, Princeton, 1987). 